\title{
A Controllable Thyristor-Based Commutation Failure Inhibitor for LCC-HVDC Transmission Systems
}

\author{
Sohrab Mirsaeidi, Member, IEEE, Dimitrios Tzelepis, Member, IEEE, \\ Jinghan He, Fellow, IEEE, Xinzhou Dong, Fellow, IEEE, \\ Dalila Mat Said, Senior Member, IEEE, and Campbell Booth
}

\begin{abstract}
Commutation failure is a serious malfunction in linecommutated High Voltage Direct Current (HVDC) converters which is mainly caused by the inverter ac faults, and results in a temporary interruption of transmitted power and damage to the converter equipment. In this paper, a Controllable Commutation Failure Inhibitor (CCFI) is developed which obviates the main drawbacks of the existing power-electronic-based and faultcurrent-limiting-based strategies. Under normal circumstances, the developed CCFI improves the steady-state stability and the power transfer capability of the inverter ac lines, while it does not cause excessive voltage stress on the converter valves. In addition, it would reduce the risk of commutation failure occurrence, since it does not lead to any voltage drop in the commutation circuit. When a fault occurs at one of the inverter ac systems, its corresponding CCFI limits the fault current depending on the reduced extinction angle. This would not only inhibit the successive commutation failures on the HVDC converter, but also extend the lifetime of components in the inverter ac systems. The practical feasibility of the developed CCFI is assessed through laboratory testing, using real-time Opal-RT hardware prototyping platform. The obtained results indicate that the developed CCFI can reliably inhibit the commutation failures during various types of faults.
\end{abstract}

Index Terms-Hybrid ac/dc power grids, HVDC transmission, line-commutated converters, and commutation failure.

\section{INTRODUCTION}

Line-Commutated Converter based HVDC (LCC-HVDC) technology has been extensively utilized around the world for long-distance bulk-power transmission due to its merits such as the thyristor's superior power handling capability

The present article outlines the results of a collaborative work conducted between Beijing Jiaotong University, Beijing, China and University of Strathclyde, Glasgow, UK.

This work was supported in part by the National Key Research and Development Plan of China (2018YFB0904602), in part by the Fundamental Research Funds for the Central Universities (2019RC051), and in part by the PHOENIX Project U.K. (SPTEN03).

S. Mirsaeidi (corresponding author) and J. He are with the School of Electrical Engineering, Beijing Jiaotong University, Beijing, People's Republic of China. (e-mail: msohrab@bjtu.edu.cn; jhhe@bjtu.edu.cn).

D. Tzelepis and C. Booth are with the Department of Electronic and Electrical Engineering, University of Strathclyde, Glasgow, United Kingdom. (e-mail: dimitrios.tzelepis@strath.ac.uk; campbell.d.booth@strath.ac.uk).

$\mathrm{X}$. Dong is with the Department of Electrical Engineering, Tsinghua University, Beijing, People's Republic of China. (e-mail: xzdong@mail.tsinghua.edu.cn).

D. M. Said is with the Centre of Electrical Energy Systems, Faculty of Electrical Engineering, Universiti Teknologi Malaysia, Johor, Malaysia. (email: dalila@utm.my). and lower operating power losses [1], [2]. Nevertheless, the development of LCC-HVDC systems suffers from some wellknown challenges such as poor voltage regulation ability and vulnerability to commutation failures during inverter ac fault incidents, which can lead to a temporary cessation of transmitted power, overheating of the valves, and misoperation of the protective relays [3].

Commutation failures are frequent dynamic incidents which have been recorded in several existing LCC-HVDC projects around the world [4]. They would become more problematic when several HVDC links terminate in one ac system such as concurrent commutation failures and forced blocking of five converter stations resulting from an inverter ac fault in South China Power Grid in 2010. This accident led to a drastic frequency reduction in the inverter ac system and overload of the adjacent HVAC lines. Also, the generators at the rectifier side were tripped and spinning reserves were activated at the inverter side to compensate for the loss of active power transfer [5]. Accordingly, commutation failure elimination has been extensively studied over the decades and a large number of approaches have been proposed. These approaches can be classified into three main categories, i.e. modification of the HVDC control system, deployment of power-electronic-based methods, and fault-current-limiting-based techniques.

For the approaches based on modification of the HVDC control system, it is pointed out by [6], [7] that the commutation failure cannot be entirely eliminated if the fault takes place very close to the inverter station. Therefore, the main targets of these approaches are to either reduce the probability of commutation failures or to expedite the HVDC system recovery after the commutation failure. The most commonly used method in this group is to immediately advance the applied firing angle to the converter thyristors after an inverter ac fault occurrence so that the commutation margin is enlarged. The main differences between the approaches of this group are: (i) the technique of detecting faults such as using symmetrical components [8] or power component fault detection method [9], (ii) the method of determining the desired firing angle such as direct measurement of commutation margin using the waveforms of the anodecathode valve voltages [8] or deployment of fuzzy-logic-based methods [10], [11], (iii) accuracy in calculation of firing angle advancement which results from considering/neglecting some of the commutation failure influencing factors including $\mathrm{dc}$ 
current [6], ac voltage, commutation inductance [8], [12], phaseangle shift, fault severity [13], and initial fault voltage angle [14], and (iv) the execution speed of firing angle advancer. However, the effectiveness of such methods is highly dependent on the fault initiation time, because if the fault occurs at the beginning or during the commutation process, the commutation failure cannot be avoided. Moreover, it is identified in [15] that advancing the firing angle augments the consumed reactive power by the converter and will further increase the inverter ac bus voltage drop. As a result, considering a limit for the extinction angle enhancement in these approaches would be necessary. In [5], a dc predictive control algorithm is developed by modifying the rectifier control system, in which the dc current order is reduced after the detection of an ac voltage disturbance. However, concerning the long distance of the HVDC lines, changing the current order at the rectifier station may not be rapid enough to deal with the commutation failure. References [16]-[20] propose a Voltage-Dependent CurrentOrder Limit (VDCOL) strategy to safeguard the HVDC system against commutation failures by limiting the current order according to the ac voltage or dc voltage. However, the variation of dc current is not considered in these studies and there is some room for further improvement [21].

Among the power-electronic-based methods, the most wellknown one is to employ Capacitor-Commutated Converters (CCCs) with fixed capacitors between the thyristor valves and the converter transformer [22]. The capacitors contribute to enhance the magnitude of commutating voltages and provide a larger commutation margin so that the commutation failure is inhibited. In addition, they improve the power factor of the inverter ac system through the reduction of reactive power consumption. However, as identified by [23], the insertion of large commutation capacitors leads to the significant voltage stress on the valves (typically 2 p.u. to 3 p.u.) during the normal operation of the converter which shortens its lifetime. Moreover, there is a possibility of ferroresonance occurring in the circuit formed by the ac system, commutation capacitors, and converter transformer. In [22], an alternative configuration, referred to as Controlled Series Capacitor Converter (CSCC), is introduced where the series capacitors are inserted between the inverter ac bus and the inverter ac system. In the CSCC configuration, even though the capacitor values can be adjusted similar to the Thyristor Controlled Series Compensation (TCSC) schemes, the controllability of capacitors is only used for the prevention of ferroresonance challenge.

The aim of the proposed methods in the third category is to prevent the commutation failure through ac voltage drop compensation by suppressing the fault current magnitude. The most popular method in this group is to use Superconducting Fault Current Limiters (SFCLs). In [24], the effectiveness of SFCL on commutation failure mitigation is qualitatively studied, while authors of [25] use a flux coupling-type SFCL to reduce successive commutation failures of the HVDC system. However, since the SFCLs operate based on a quenching characteristic, they are not controllable and have not the ability to suppress the fault current proportional to the reduced extinction angle. In order to remedy this challenge, reference [26] develops a controllable Commutation Failure Prevention Module (CFPM), in which the fault current is suppressed to a desired value based on the fault intensity. Nevertheless, in the developed module, a large isolation transformer and an extra three-phase diode bridge are required which significantly increase its capital cost and power losses. In addition, the voltage drop caused by the inductor of the CFPM circuit under normal operating conditions enhances the probability of commutation failure.

This paper attempts to overcome the main challenges of the above-mentioned strategies through the development of a Controllable Commutation Failure Inhibitor (CCFI). Indeed, at no-fault conditions, the proposed CCFI acts similar to a CSCC, except that it does not cause excessive voltage drop on the inverter switches, because a significant portion of its capacitance is eliminated by a series inductor. Moreover, in spite of the fault-current-limiting-based strategies, it is fully controllable and does not cause any voltage drop in the commutation circuit under normal conditions. In case a fault occurs at one of the receiving ac systems, its associated CCFI behaves like a fault current limiter to inhibit the occurrence of commutation failure at the inverter station.

The remainder of this paper is organized as follows: Section II describes the commutation process and mechanism of commutation failure in line-commutated converters; in Section III, the structure of the proposed CCFI is presented and its operating principles are theoretically analyzed; in Section IV, the test network is introduced and the practical feasibility of the proposed CCFI is validated through laboratory testing; and finally, concluding remarks are given in Section V.

\section{Commutation Process and Mechanism of COMMUTATION FAILURE}

Fig. 1 depicts the basic structure of a six-pulse converter at the inverter side which is referred to as Graetz bridge. The term six-pulse is due to the six commutations of switching operations per period, which forms a characteristic harmonic ripple of six times the fundamental frequency in the dc voltage. The Graetz bridge includes six thyristor valves, $T_{1 i}$ to $T_{6 i}$, which are numbered according to the sequence they are triggered. At any instant, two valves are conducting, one from the upper group of valves and second from the lower group. Fig. 2 displays the waveforms of the dc voltage and the currents through valves $T_{1 i}$ to $T_{6 i}$ for the inverter shown in Fig. 1. As can be seen from the figure, under steady-state conditions, each valve conducts for 120 degrees and the interval between consecutive firing pulses is 60 degrees. The instantaneous line-to-neutral voltages of each phase, $e_{k}(k=a, b, c)$, are expressed as:

$$
\begin{aligned}
& e_{a}=\sqrt{2} E_{L L} \cos \left(\omega t+60^{\circ}\right) \\
& e_{b}=\sqrt{2} E_{L L} \cos \left(\omega t-60^{\circ}\right) \\
& e_{c}=\sqrt{2} E_{L L} \cos \left(\omega t-180^{\circ}\right)
\end{aligned}
$$

where $E_{L L}$ is the rms phase-to-phase ac voltage, and $\omega$ is the angular frequency in radians. The switching of current conduction from one of the thyristor valves to another in the same row of a converter bridge is referred to as commutation. The highlighted blue loop in Fig. 1 shows the electrical circuit for commutation from valve $T_{1 i}$ to valve $T_{3 i}$. In this case, firing 
angle, $\alpha_{i}$, corresponds to the time when valve $T_{3 i}$ is fired after the commutation voltage $\left(e_{b}-e_{a}\right)$ has turned positive. Due to the inductance of the converter transformer and its connected ac system, commutation process cannot be instantaneous and takes for a certain time, in which both valves $T_{1 i}$ and $T_{3 i}$ conduct. The angle corresponding to this time duration is termed as overlap angle, $\mu_{i}$. After the overlap time, a reverse voltage requires to be applied across valve $T_{1 i}$ for a certain duration. This would remove the charges stored during its conduction process such that it can withstand a voltage in the forward direction. This negative voltage is applied during the time corresponding to the extinction angle, $\gamma_{i}$. The voltage equation during the commutation from valve $T_{1 i}$ to valve $T_{3 i}$ can be written as:

$$
e_{b}-e_{a}=\omega L_{c} \frac{\mathrm{d} i_{3}}{\mathrm{~d} \omega t}-\omega L_{c} \frac{\mathrm{d} i_{1}}{\mathrm{~d} \omega t}
$$

where $i_{1}$ and $i_{3}$ are instantaneous currents flowing through valves $T_{1 i}$ and $T_{3 i} ; L_{c}$ is the commutation reactance of each phase; and, $I_{d}$ is the dc current. Substituting (1) in (2), and using $i_{1}=I_{d}-i_{3}$ gives:

$$
\sqrt{2} E_{L L} \sin (\omega t)=\omega L_{c} \frac{\mathrm{d} i_{3}}{\mathrm{~d} \omega t}-\omega L_{c} \frac{\mathrm{d}\left(I_{d}-i_{3}\right)}{\mathrm{d} \omega t}
$$

Considering $\mathrm{d} I_{d} / \mathrm{d} \omega t=0$, Eq. (3) becomes:

$$
\int_{0}^{I_{d}} 2 \omega L_{c} \mathrm{~d} i_{3}=\int_{\alpha_{i}}^{\pi-\gamma_{i}} \sqrt{2} E_{L L} \sin (\omega t) \mathrm{d} \omega t
$$

where $\alpha_{i}$ and $\gamma_{i}$ are respectively firing angle and extinction angle of the thyristor valves at the inverter station. Therefore, $I_{d}$ can be computed as:

$$
I_{d}=\frac{\sqrt{2} E_{L L}}{2 \omega L_{c}}\left(\cos \alpha_{i}+\cos \gamma_{i}\right)
$$

In case of a fault incident at the inverter ac side, the ac voltage magnitudes of the affected phases decrease which results in a dc voltage drop according to the principles of ac/dc conversion. Such a reduction in the dc voltage increases $I_{d}$ in order to sustain the active power at the rated power of inverter station. Accordingly, the overlap angle also increases which leads to a reduction in the extinction angle. When $\gamma_{i}$ drops below the thyristor turn-off time, leads to the unexpected turn-on of $T_{1 i}$ supposed to be off, and a commutation failure takes place. In the next scheduled commutation, $T_{4 i}$ is also fired which creates a dc short-circuit, since both valves of a converter arm simultaneously conduct. This leads to a zero dc voltage across the faulty converter arm, and hence no active power can be transmitted through it [27]-[29].

\section{Proposed Controllable Commutation Failure INHIBITOR (CCFI)}

\section{A. Structure and Operating Principles of the Proposed CCFI}

Fig. 3 demonstrates the structure of the proposed CCFI in a hybrid ac/dc grid including one dc line and two receiving ac systems. As can be seen from the figure, it is composed of a

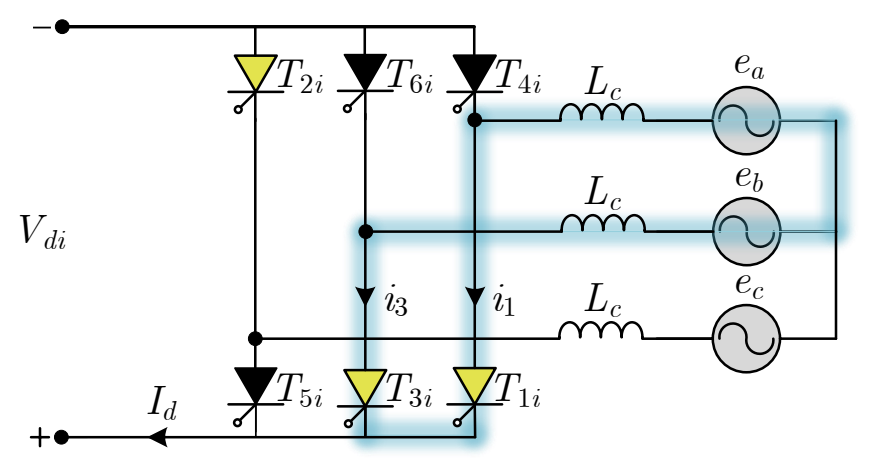

Fig. 1: Graetz bridge.

thyristor-controlled inductor in series with a capacitor which operates as a double-function device by applying different firing angles to the thyristor valves. Under normal conditions, the CCFI behaves similar to a CSCC with a small capacitance which enhances the magnitude of the inverter commutating voltages and provides a larger commutation margin, while it does not cause excessive voltage stress on the inverter valves. In addition, it improves the system steady-state stability and reduces the transmission losses in the receiving ac systems. When a fault occurs at one of the inverter ac systems, its corresponding CCFI limits the fault current depending on the reduced extinction angle. This would not only prevent the commutation failure in the inverter station, but also extend the lifespan of components in the inverter ac systems.

Fig. 4 depicts the schematic diagram of CCFI1 control system shown in Fig. 3. According to the figure, fault occurrence at the inverter ac system 1 is recognized by either symmetrical or non-symmetrical fault detector, depending on the fault type. In the non-symmetrical fault detector, the negative-sequence voltage is used as fault detection criterion, since it is the only sequence which appears in all types of non-symmetrical faults, i.e. single-line-to-ground, line-to-line, and line-to-line-to-ground faults. Once the measured negativesequence voltage exceeded a pre-determined threshold value, $\left(v_{2}\right)_{t h r}$, signal $f l t_{n o n-S y m}$ is issued and a non-symmetrical fault is recognized.

However, for detection of symmetrical faults, $a b c-\alpha \beta$ transformation is applied. The idea of employing this transformation is that the magnitude of rotating vector $v_{\alpha \beta}$, $\left|v_{\alpha \beta}\right|=\sqrt{v_{\alpha}^{2}+v_{\beta}^{2}}$, is a dc quantity for symmetrical threephase voltages. In the developed CCFI, when a three-phase fault occurs, $\left|v_{\alpha \beta}\right|$ is compared with its filtered signal, $\left|v_{\alpha \beta}\right|_{f i l}$, which is considered as the pre-fault voltage. If $\left|v_{\alpha \beta}\right|_{\text {dif }}=$ $\left|v_{\alpha \beta}\right|_{f i l}-\left|v_{\alpha \beta}\right|$ is greater than a pre-defined threshold, then the symmetrical fault detector output is activated.

In case of a fault detection at inverter ac system 1, by either symmetrical or non-symmetrical fault detector, CCFI1 is transferred to its fault current limiting mode through a multiplexer. Accordingly, a suitable firing angle is applied to CCFI1 valves depending on the reduced extinction angle to limit the fault current and inhibit the commutation failure. 


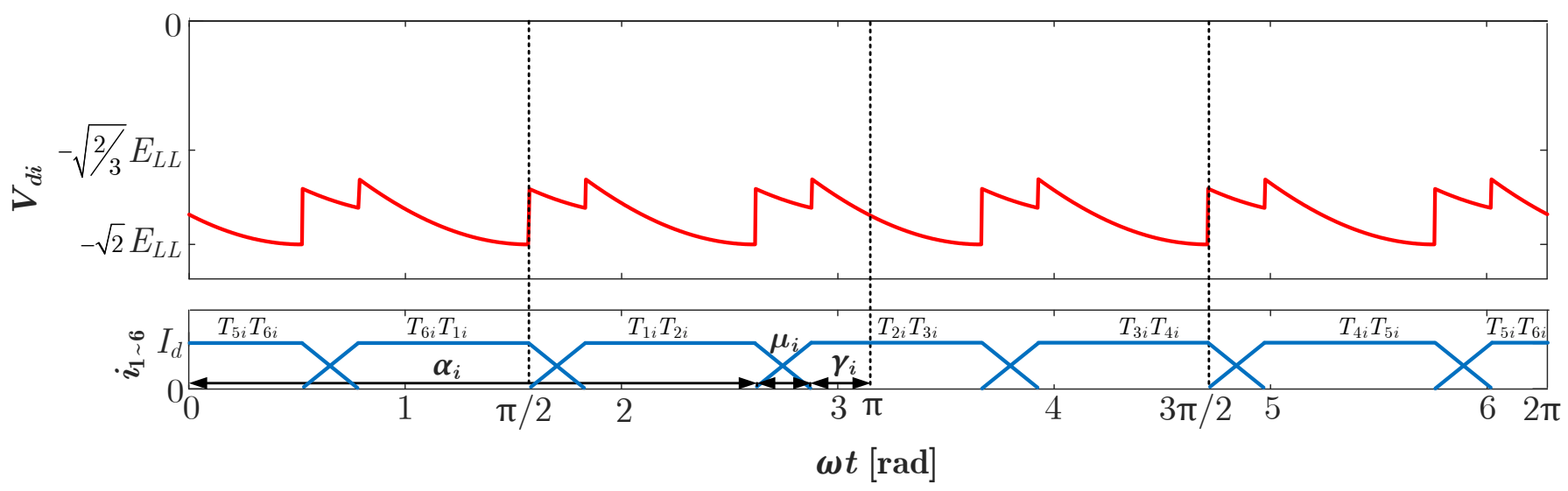

Fig. 2: Waveforms of the dc voltage and the currents through valves $T_{1 i}$ to $T_{6 i}$ for the inverter shown in Fig. 1 .

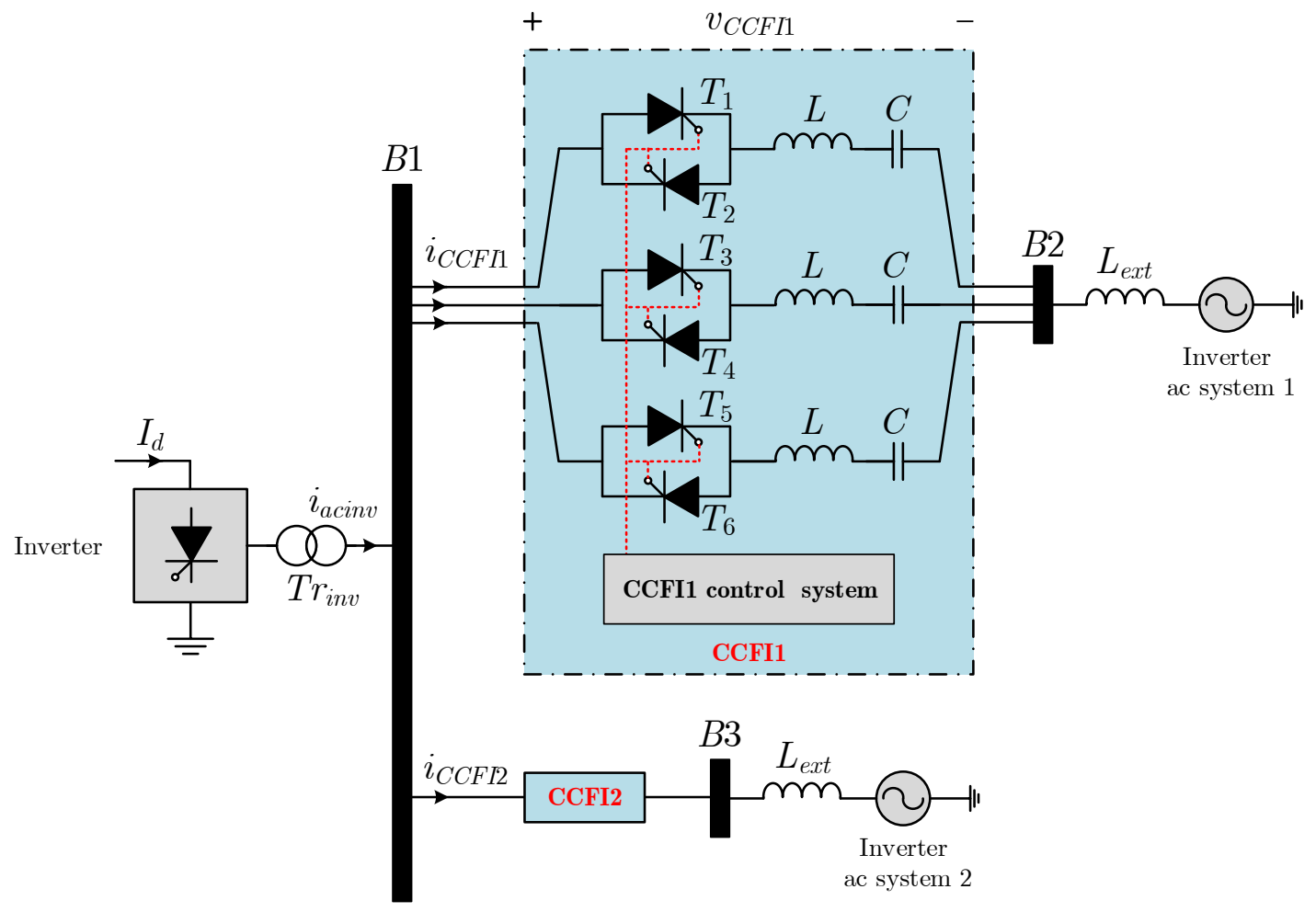

Fig. 3: Structure of the proposed CCFI in a hybrid ac/dc grid including one dc line and two receiving ac systems.

\section{B. CCFI Operation Analysis}

Fig. 5 depicts the phase voltage and current waveforms of CCFI1 shown in Fig. 3 during steady-state conditions. In this figure, the time reference, termed as "Original Reference (OR)" is taken at the positive-going zero-crossing of $v_{C C F I 1}$. However, for the simplicity of analysis, a Shifted Reference (SR) is considered which is taken when thyristor $T_{1}$ starts to conduct. Accordingly, $v_{C C F I 1}$ in terms of the shifted reference can be expressed as:

$$
\begin{aligned}
v_{C C F I 1, S R} & =V_{p} \sin (\omega t-\sigma) \\
& =V_{p} \sin (\omega t) \cos \sigma-V_{p} \cos (\omega t) \sin \sigma
\end{aligned}
$$

The voltage equation across CCFI1 circuit can be written as:

$$
v_{C C F I 1, S R}=v_{L, S R}+v_{C, S R}
$$

Substituting (6) in (7) and taking Laplace transform results in:

$$
\begin{aligned}
& V_{p} \cos \sigma\left(\frac{\omega}{s^{2}+\omega^{2}}\right)-V_{p} \sin \sigma\left(\frac{s}{s^{2}+\omega^{2}}\right)= \\
& \left(L s I_{L, S R}(s)-L i_{L, S R}(0)\right) \\
& +\left(\frac{1}{C s} I_{C, S R}(s)+\frac{1}{s} v_{C, S R}(0)\right)
\end{aligned}
$$




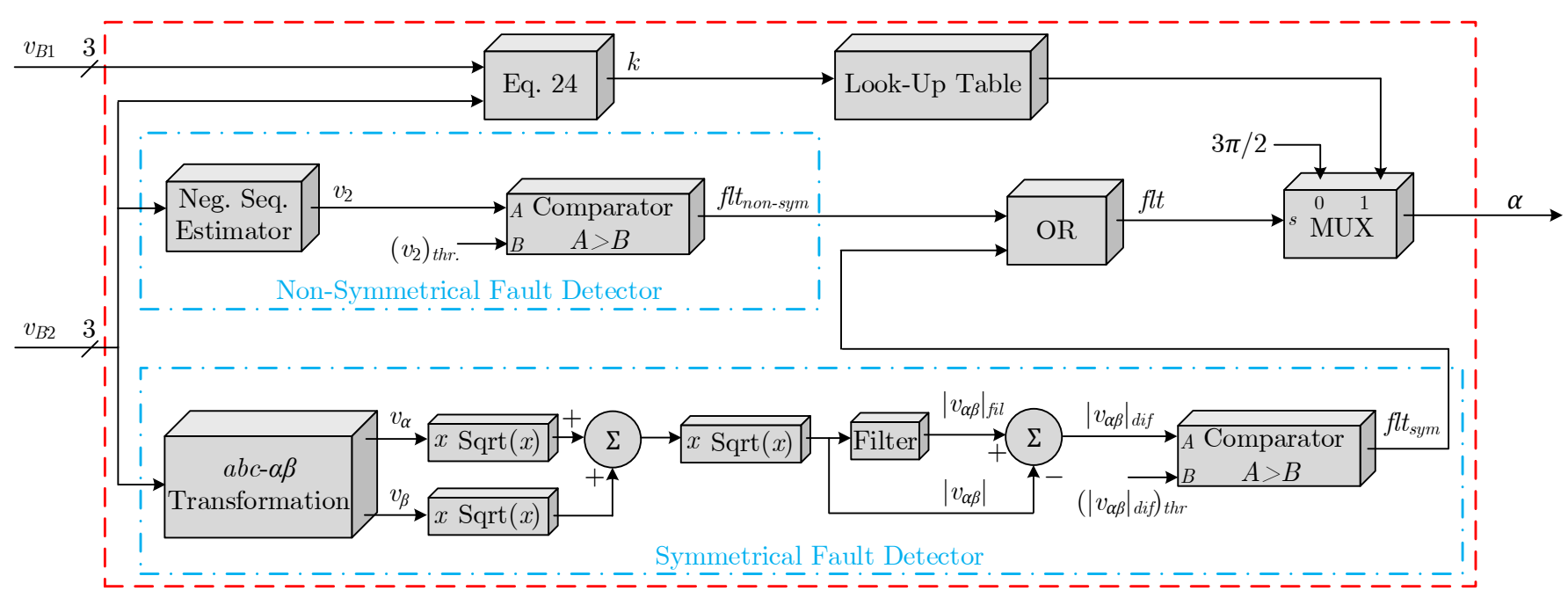

Fig. 4: Schematic diagram of CCFI1 control system.

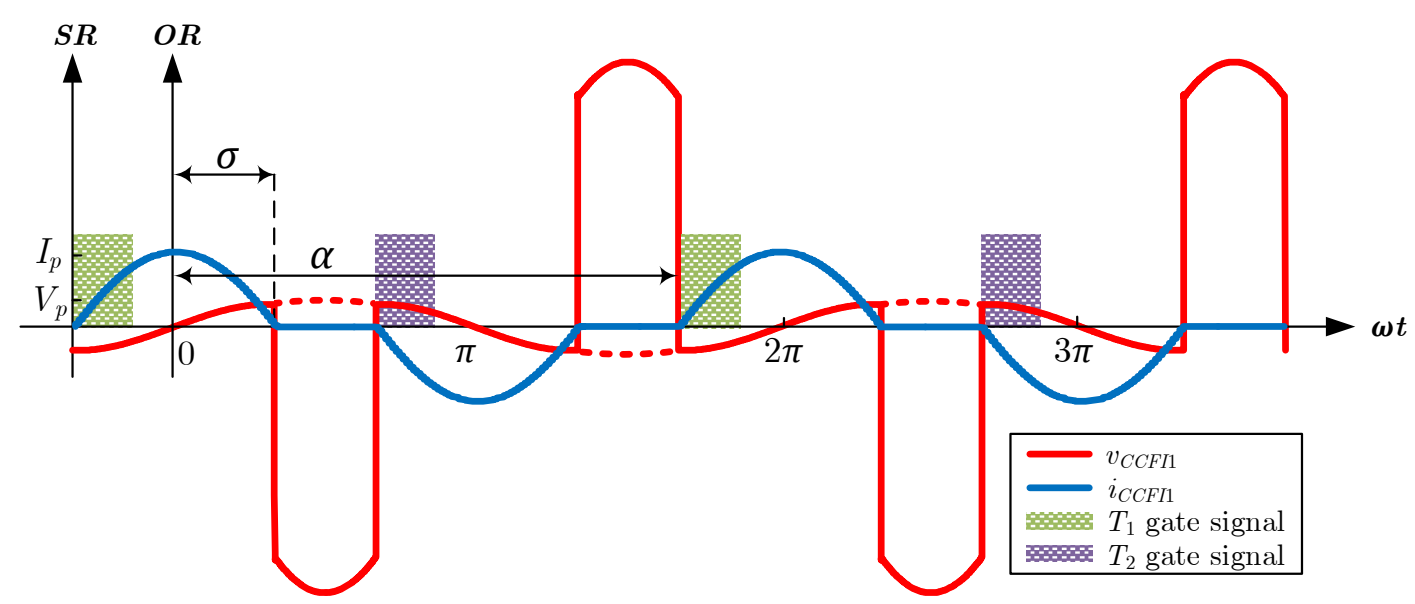

Fig. 5: Phase voltage and current waveforms of CCFI1.

Since $L$ and $C$ are connected in series, $I_{C C F I 1, S R}(s)=$ $I_{L, S R}(s)=I_{C, S R}(s)$. Also, $i_{L, S R}(0)=0$ according to Fig. 5 . Therefore, $I_{C C F I 1, S R}(s)$ can be decribed as:

$$
\begin{aligned}
I_{C C F I 1, S R}(s)= & V_{p} \omega \cos \sigma L \frac{s}{\left(s^{2}+\omega^{2}\right)\left(s^{2}+\omega_{0}^{2}\right)} \\
& -\frac{V_{p} \sin \sigma}{L} \frac{s^{2}}{\left(s^{2}+\omega^{2}\right)\left(s^{2}+\omega_{0}^{2}\right)} \\
& -\frac{v_{C, S R}(0)}{L} \frac{1}{s^{2}+\omega_{0}^{2}}
\end{aligned}
$$

where $\omega_{0}=\frac{1}{\sqrt{L C}}$ denotes the resonant angular frequency. Eq. (9) can be expressed in the time domain by taking the Laplace inverse as:

$$
\begin{aligned}
i_{C C F I 1, S R}= & A \omega \cos \sigma\left(\cos \omega t-\cos \omega_{0} t\right) \\
& -A \sin \sigma\left(\omega_{0} \sin \omega_{0} t-\omega \sin \omega t\right) \\
& -D v_{C, S R}(0) \sin \omega_{0} t
\end{aligned}
$$

where $A=\frac{V_{p}}{L\left(\omega_{0}^{2}-\omega^{2}\right)}$ and $D=\frac{1}{L \omega_{0}}$. Simplifying (10) results in:

$$
\begin{aligned}
i_{C C F I 1, S R}= & A \omega \cos (\omega t-\sigma)-A \omega \cos \sigma \cos \omega_{0} t \\
& -\left(A \omega_{0} \sin \sigma+D v_{C, S R}(0)\right) \sin \omega_{0} t
\end{aligned}
$$

$i_{C C F I 1}$ in terms of the original time reference for the range of $[-\sigma, \sigma]$ can be obtained by adding $\sigma / \omega$ to the time variable in (11) which results in:

$$
\begin{aligned}
i_{C C F I 1}= & A \omega \cos \omega t-(A \omega \cos \sigma \cos \bar{\omega} \sigma \\
& \left.+A \omega_{0} \sin \sigma \sin \bar{\omega} \sigma+D v_{C, S R}(0) \sin \bar{\omega} \sigma\right) \cos \omega_{0} t \\
& +\left(A \omega \cos \sigma \sin \bar{\omega} \sigma-A \omega_{0} \sin \sigma \cos \bar{\omega} \sigma\right. \\
& \left.-D v_{C, S R}(0) \cos \bar{\omega} \sigma\right) \sin \omega_{0} t
\end{aligned}
$$

where $\bar{\omega}=\omega_{0} / \omega$. As can be seen from Fig. 5, in the steadystate conditions, $i_{C C F I 1}$ is an even function, and hence the coefficient of $\sin \omega_{0} t$ in (12) takes a value of zero. Therefore, $v_{C, S R}(0)$ can be calculated by: 


$$
v_{C, S R}(0)=\frac{A}{D} \omega \cos \sigma \frac{\sin \bar{\omega} \sigma}{\cos \bar{\omega} \sigma}-\frac{A}{D} \omega_{0} \sin \sigma
$$

By substituting (13) into (12) and simplifying:

$$
i_{C C F I 1}=A \omega \cos \omega t-\frac{A \omega \cos \sigma}{\cos \bar{\omega} \sigma} \cos \bar{\omega} \omega t
$$

As can be seen from Fig. 5, $i_{C C F I 1}$ has even and quarterwave symmetry. Therefore, its Fourier series can be written as:

$$
i_{C C F I 1}=\sum_{n} I_{C C F I 1(n)} \cos n \omega t
$$

where,

$$
I_{C C F I 1(n)}= \begin{cases}0 & \text { for } n \text { even } \\ \frac{4}{\pi} \int_{0}^{\pi / 2} i_{C C F I 1} \cos n \omega t \mathrm{~d} \omega t & \text { for } n \text { odd }\end{cases}
$$

As a result, the rms value of the fundamental frequency component of $i_{C C F I 1}$ can be calculated by substituting (14) in (16):

$$
\begin{aligned}
I_{C C F I 1(1), r m s}= & \frac{A \omega}{\pi \sqrt{2}}(2 \sigma+\sin 2 \sigma) \\
& -\frac{4 A \omega}{\pi \sqrt{2}\left(\bar{\omega}^{2}-1\right)}\left(\bar{\omega} \cos ^{2} \sigma \tan \bar{\omega} \sigma-\sin \sigma \cos \sigma\right)
\end{aligned}
$$

Substituting $A=\frac{V_{p}}{L\left(\omega_{0}^{2}-\omega^{2}\right)}=\frac{C V_{p} \bar{\omega}^{2}}{\bar{\omega}^{2}-1}$ and $\sigma=2 \pi-\alpha$ in (17) results in:

$$
\begin{aligned}
I_{C C F I 1(1), r m s}= & \frac{V_{C C F I 1, r m s} C \omega \bar{\omega}^{2}}{\left(\bar{\omega}^{2}-1\right)}\left[\frac{2(2 \pi-\alpha)}{\pi}\right. \\
& +\frac{\sin 2(2 \pi-\alpha)}{\pi} \\
& -\frac{4 \bar{\omega} \cos ^{2}(2 \pi-\alpha) \tan \bar{\omega}(2 \pi-\alpha)}{\pi\left(\bar{\omega}^{2}-1\right)} \\
& \left.+\frac{4 \sin (2 \pi-\alpha) \cos (2 \pi-\alpha)}{\pi\left(\bar{\omega}^{2}-1\right)}\right]
\end{aligned}
$$

where $V_{C C F I 1, r m s}=V_{p} / \sqrt{2}$. The impedance magnitude of the LC circuit in CCFI1 can be determined as:

$$
\left|Z_{L C}\right|=\frac{1}{\omega C}-\omega L=\frac{1-\frac{\omega^{2}}{\omega_{0}^{2}}}{\omega C}=\frac{\bar{\omega}^{2}-1}{C \omega \bar{\omega}^{2}}
$$

Substituting (19) in (18) results in:

$$
I_{C C F I 1(1), r m s}=\frac{k V_{C C F I 1, r m s}}{\left|Z_{L C}\right|}
$$

where $k$ is the term inside the square bracket of Eq. (18). For any given thyristor firing angle, $\alpha$, the fundamental frequency component of CCFI1 impedance magnitude can be obtained using (20) as follows:

$$
Z_{C C F I 1(1)}=\frac{V_{C C F I 1, r m s}}{I_{C C F I 1(1), r m s}}=\frac{\left|Z_{L C}\right|}{k}
$$

Fig. 6 shows the relationship between parameter $k$ and CCFI1 thyristor firing angle considering $\bar{\omega}=3$, at which symmetry of current waveform is preserved and the thyristor arms are equally stressed. Under normal conditions, the thyristor firing angle is $3 \pi / 2$, and $k$ takes the value of 1 . Accordingly, $Z_{C C F I 1(1)}$ becomes equivalent to the LC circuit impedance magnitude, $\left|Z_{L C}\right|$. Hence, by considering $X_{C}>X_{L}$, the power system stability and power transfer capability can be improved at nofault conditions. In case of a fault incident, CCFI1 thyristor firing angle is selected in the range of $6.28<\alpha<6.72$ radians depending on the reduced extinction angle, to limit $I_{C C F I 1(1), r m s}$ and inhibit the commutation failures. It shall be noted that the insertion of any type of Fault Current Limiter (FCL) decreases the magnitude of short-circuit current and may lead to the misoperation of protective relays, since it affects the admittance matrix of the network. However, it is a common practice that the influenced relays are re-adjusted and re-coordinated with each other after the installation of an FCL based on the maximum limited current by the FCL. As a result, readjustment of the relays located on each inverter ac system after implemenation of the proposed strategy would be necessary.

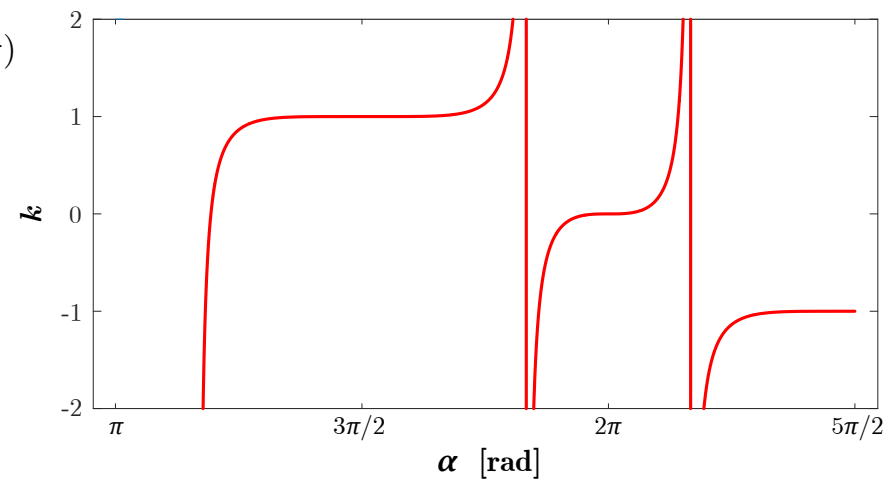

Fig. 6: Relationship between parameter $k$ and CCFI1 thyristor firing angle.

Neglecting the commutation overlap for the inverter shown in Fig. 3, the rms value of the fundamental frequency component of inverter ac current can be calculated using Fourier analysis as:

$$
I_{i n v(1), r m s}=\frac{1}{\pi \sqrt{2}} \int_{-\pi}^{\pi} I_{d} \cos \omega t \mathrm{~d} \omega t=\frac{\sqrt{6}}{\pi} I_{d}
$$

By substituting (5) in (22) and considering $I_{C C F I 1(1), r m s}=$ $m I_{i n v(1), r m s}$, where $m$ is a constant between 0 and 1 , $I_{C C F I 1(1), r m s}$ can be written as:

$$
I_{C C F I 1(1), r m s}=\frac{m \sqrt{3} E_{L L}}{\pi \omega L_{c}}\left(\cos \alpha_{i}+\cos \gamma_{i}\right)
$$


By equating (18) and (23), and considering a minimum commutation margin, $\gamma_{\text {min }}$, parameter $k$ is obtained as:

$$
k=\frac{m \sqrt{3} E_{L L}\left(\bar{\omega}^{2}-1\right)\left(\cos \alpha_{i}+\cos \gamma_{\text {min }}\right)}{\pi L_{c} C \omega^{2} \bar{\omega}^{2} V_{C C F I 1, r m s}}
$$

As discussed before, under normal conditions, CCFI1 is used to compensate for the inductive reactance of its corresponding ac line. The resonant angular frequency of the compensated ac line by CCFI1 is expressed as:

$$
\omega_{0, t o t}=\omega \underbrace{\sqrt{k_{s e r}}}_{\bar{\omega}_{t o t}}=\omega \sqrt{\frac{X_{C}}{X_{L_{e x t}}+X_{L}}}=\frac{1}{\sqrt{C\left(L_{e x t}+L\right)}}
$$

where $k_{\text {ser }}$ is the compensation degree of the compensated ac line which is typically in the range of $25 \%-70 \%$ [30]; $L$ and $C$ are CCFI1 parameters; and, $L_{e x t}$ is the equivalent series inductance connected to CCFI1. It shall be noted that even though the practical upper limit of $k_{s e r}$ is $70 \%$, in the proposed strategy, in order to prevent the excessive voltage stress on the inverter valves, only $30 \%$ of the ac line is compensated. The resonant angular frequency of CCFI1 is $\omega_{0}=\omega \bar{\omega}=1 / \sqrt{L C}$. Therefore:

$$
C=\frac{1}{L \bar{\omega}^{2} \omega^{2}}
$$

By substituting (26) in (25), $L$ is determined as:

$$
L=\frac{k_{s e r} L_{e x t}}{\bar{\omega}^{2}-k_{\text {ser }}}
$$

The capacitance of CCFI1 is also determined by substituting (27) in (26):

$$
C=\frac{\bar{\omega}^{2}-k_{\text {ser }}}{k_{\text {ser }} \omega^{2} \bar{\omega}^{2} L_{e x t}}
$$

In order to better illustrate the selection of parameters in the proposed CCFI, let's assume $L_{\text {ext }}=16 \mathrm{mH}, \bar{\omega}=3$, and the frequency of the inverter ac system is $50 \mathrm{~Hz}$. In this case, if the series compensation degree of $25 \%$ is desired, then a $0.45 \mathrm{mH}$ inductor (corresponding to inductive reactance of $0.14 \Omega$ ) and a $2462.66 \mu \mathrm{F}$ capacitor (corresponding to capacitive reactance of $1.29 \Omega$ ) are required according to (27) and (28), respectively. While for $k_{\text {ser }}=70 \%$, the suitable CCFI parameters are $L=1.34 \mathrm{mH}$ (corresponding to inductive reactance of $0.42 \Omega$ ) and $C=834.29 \mu \mathrm{F}$ (corresponding to capacitive reactance of $3.81 \Omega$ ). As a result, the size and cost-effectiveness of the proposed CCFI depend on the degree of series compensation. In fact, the larger the degree of series compensation, the greater inductive and capacitive reactance required. However, the optimal value of $k_{\text {ser }}$ should be determined based on the voltage stress on the converter valves, and the stability and power transfer capability of the inverter ac system.

\section{EXPERIMENTAL VALIDATION}

Fig. 7 shows the single-line diagram of the test network which connects the ac system in the rectifier side to two identical ac systems in the inverter side through a monopolar $500 \mathrm{kV}$ and $1000 \mathrm{MW}$ HVDC system. The HVDC system consists of a dc line modeled by a $\mathrm{T}$ circuit, two 12-pulse converters, ac filters, and shunt capacitors in both rectifier and inverter sides.

In order to verify the effectiveness of the proposed CCFI under real-time conditions, an experimental setup has been developed. The Opal-RT Simulator is one of the most advanced real-time simulation devices which enables users to conduct high-fidelity simulations and test even the most complex systems with ease and at the lowest possible cost. Fig. 8 demonstrates the schematic diagram of the experimental test. As shown in the figure, first, the MATLAB/Simulink models are built in a host computer installed with Opal RT-Lab software. The host computer is linked to the Opal-RT simulator through Ethernet, and then the simulation results are observed in a digital storage oscilloscope via connecting probes. The entire real-time model consists of two separate subsystems, where Subsystem 1 denotes the test network, and Subsystem 2 represents the control system of CCFI1. The input signals of subsystem 2 which are produced by subsystem 1 include the three-phase voltages across CCFI1. Also, the output of subsystem 2, i.e. CCFI1 thyristor firing angle, forms the input of subsystem 1 .

The results obtained from the experimental test of the proposed CCFI under different fault types, i.e. single-lineto-ground, double-line, double-line-to-ground, and three-phase faults, are displayed in Fig. 9. In all analyzed cases, fault $\mathrm{F}$ (as indicated in Fig. 7) is applied at $t=2 \mathrm{~s}$ for a duration of 0.2 s. In this figure, $V_{d i}$ and $I_{d}$ respectively denote the measured voltage and current of the HVDC line at the inverter station; $\gamma_{i}$ represents the inverter extinction angle; $I_{C C F I 1(1), r m s}$ is the rms value of the fundamental frequency component of current flowing through CCFI1; also, $v_{2}$ and $\left|v_{\alpha \beta}\right|$ represent the negative-sequence voltage and the magnitude of rotating vector $v_{\alpha \beta}$, respectively.

As can be seen in all cases, before the fault occurrence, the inverter extinction angle has a constant value which is controlled by the HVDC control system. Also, $I_{d}=2 \mathrm{kA}$ and $V_{d i}=500 \mathrm{kV}$ which leads to the transmission of $1000 \mathrm{MW}$ active power through the HVDC line. Under such conditions, CCFI1 thyristor firing angle takes the value of $3 \pi / 2$, and hence, CCFI1 operates as a capacitor which improves the steady-state stability of its connected line.

With reference to Fig. 9(a), when a single-line-to-ground fault happens, the inverter ac voltage, and thus, the involved commutating voltages are reduced in magnitude. Such a voltage drop reduces the dc voltage, $V_{d i}$, which leads to an increase in the dc current. Since the overlap angle is proportional to the dc current, it also increases and reduces the extinction angle, leading to two consecutive commutation failures initiated at $t=2.02 \mathrm{~s}$.

In order to inhibit the successive commutation failures, CCFI1 control system is activated once the fault is detected 


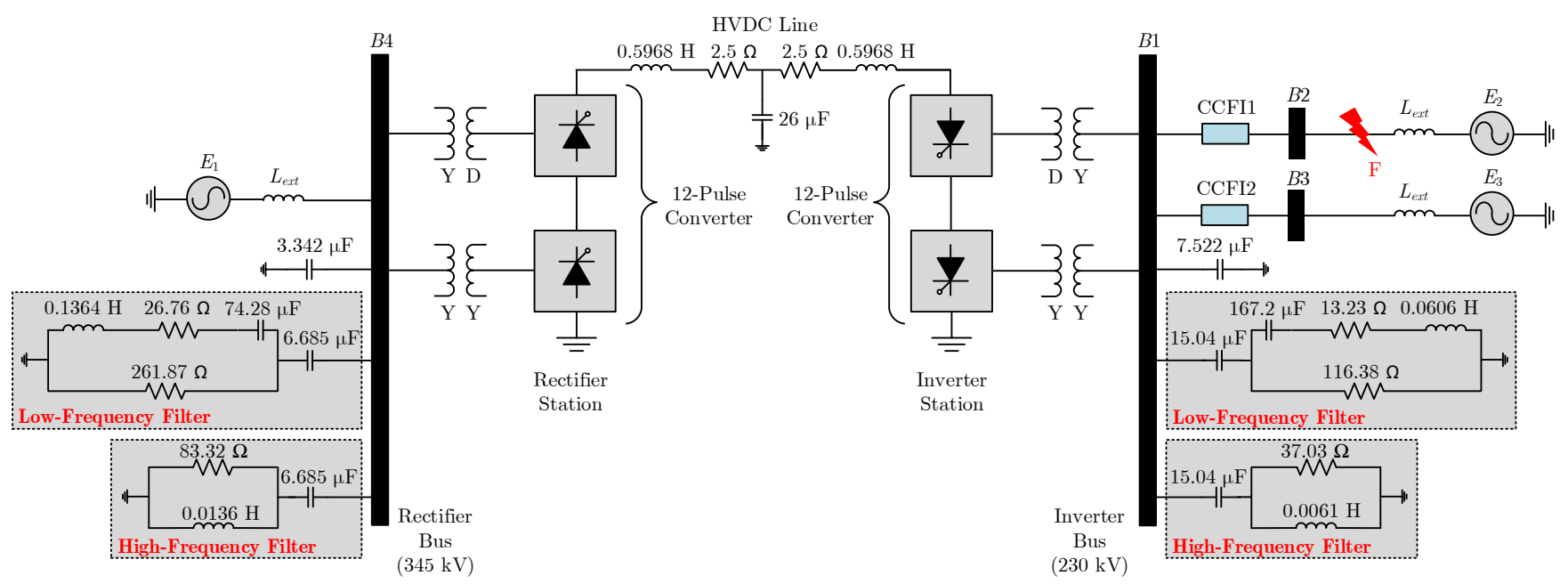

Fig. 7: Single-line diagram of the test network.

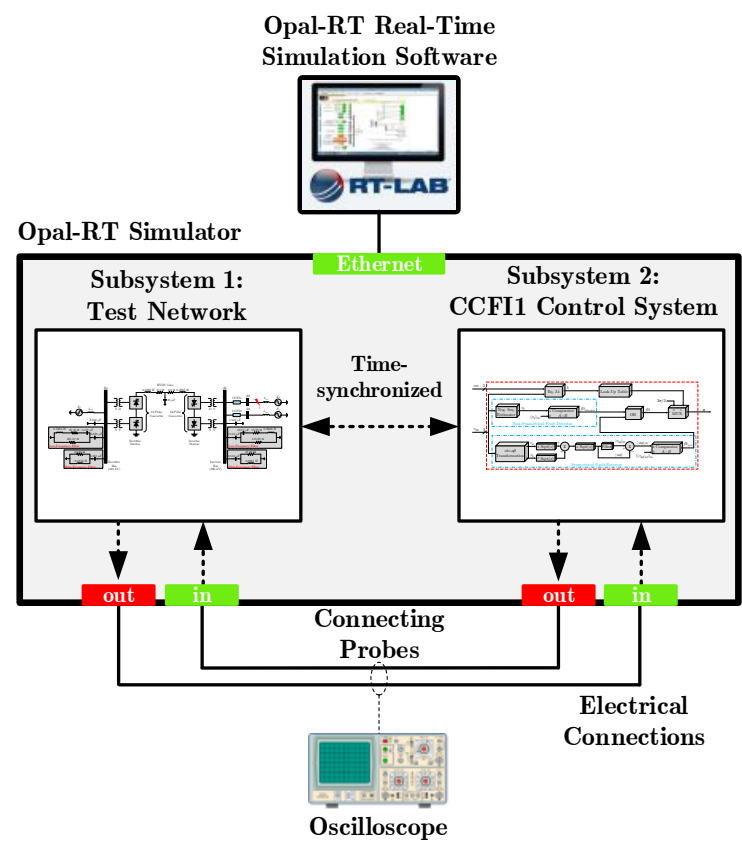

(a)

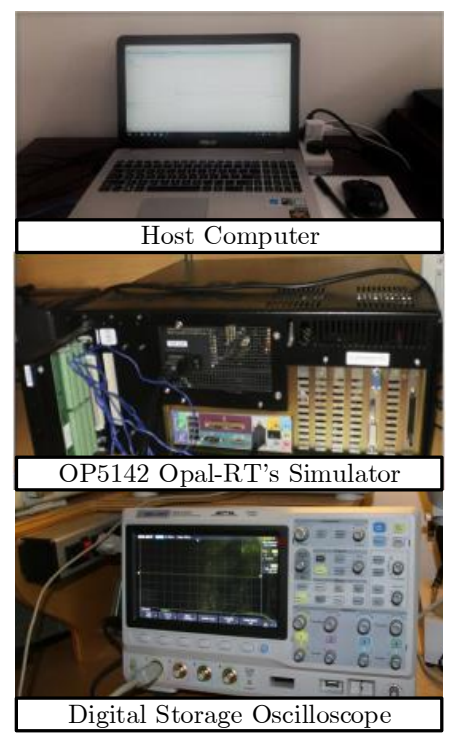

(b)

Fig. 8: Schematic diagram of the experimental test: (a) experimental setup, and (b) experimental arrangement.

by the non-symmetrical fault detector. As can be seen from Fig. 9, both harmonics and the delay associated with the negative-sequence voltage phasor measurement have influenced the voltage waveforms. In order to eliminate such challenges, the threshold values are selected such that the fault detection signal is triggered up to a maximum of $2 \mathrm{~ms}$ after the fault initiation time and the harmonic distortion does not lead to the deactivation of the CCFI1 during the fault. Subsequently, the value of $k$ is determined using Eq. (24) such that the minimum commutation margin is ensured $(k=0.22$ in this case). Finally, the corresponding value of CCFI1 thyrsitor firing angle, $\alpha=6.622$ radians, is selected using a look-up table which has been set according to Fig. 6. This leads to the limitation of the fault current up to $14.11 \mathrm{kA}$, thereby commutation failure is prevented at the inverter station. A similar analysis can be performed for other types of faults, except that $\left|V_{\alpha \beta}\right|_{\text {dif }}$ is used as a fault detection signal for the symmetrical three-phase fault.

\section{CONCLUSiON}

In this paper, a Controllable Commutation Failure Inhibitor (CCFI) has been proposed which consists of a thyritorcontrolled inductor in series with a capacitor. The developed CCFI operates as a double-function device by applying different firing angles to its thyristor switches. At no-fault conditions, the CCFI improves the steady-state stability and power transfer 

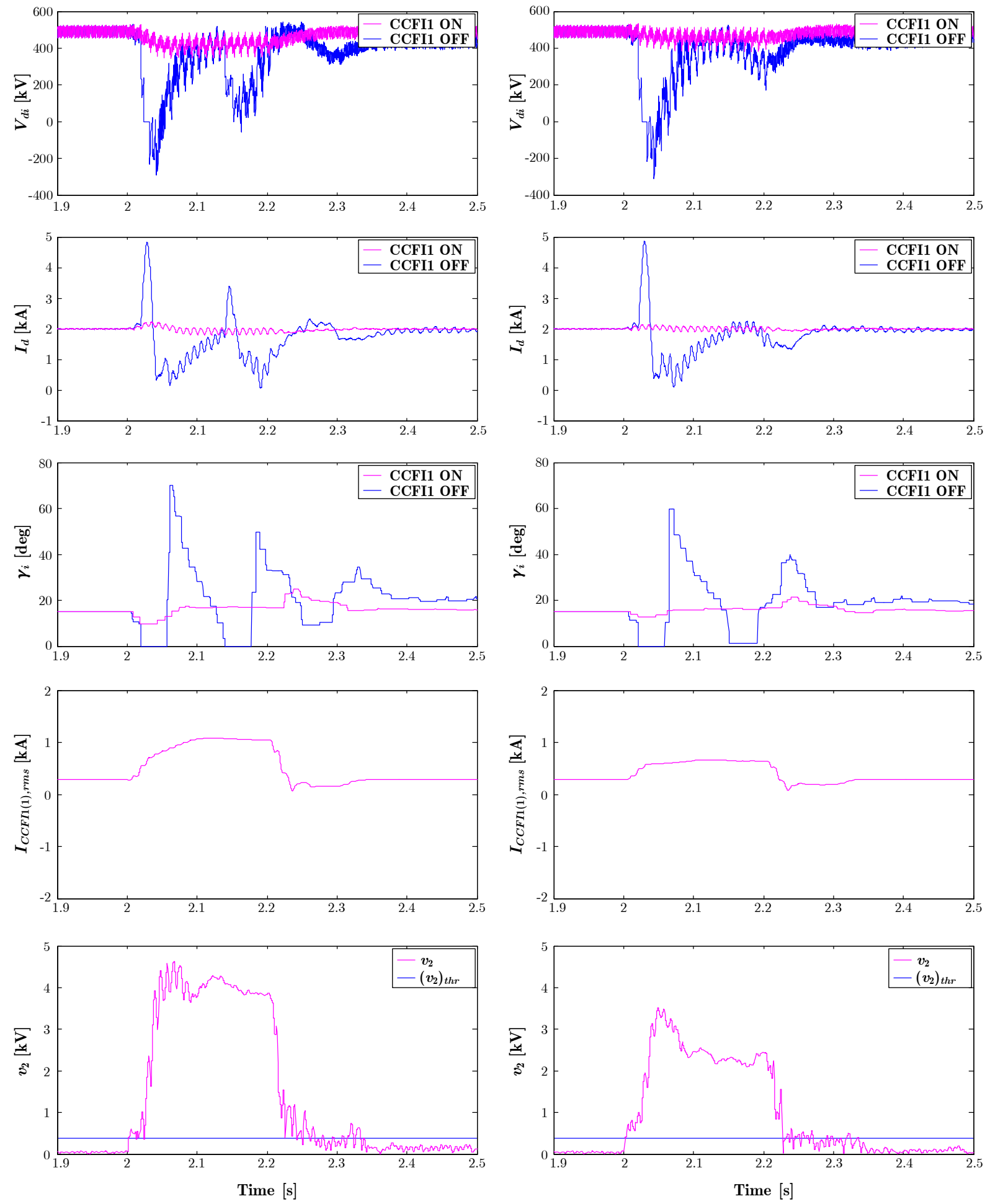

(a)

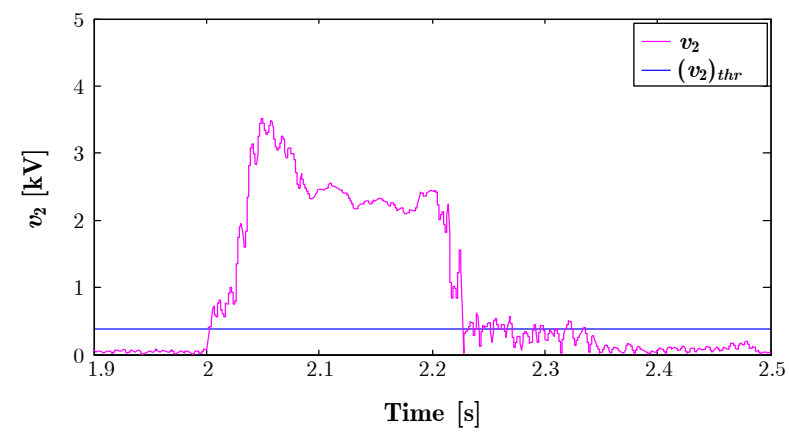

(b)

Fig. 9: To be continued. 

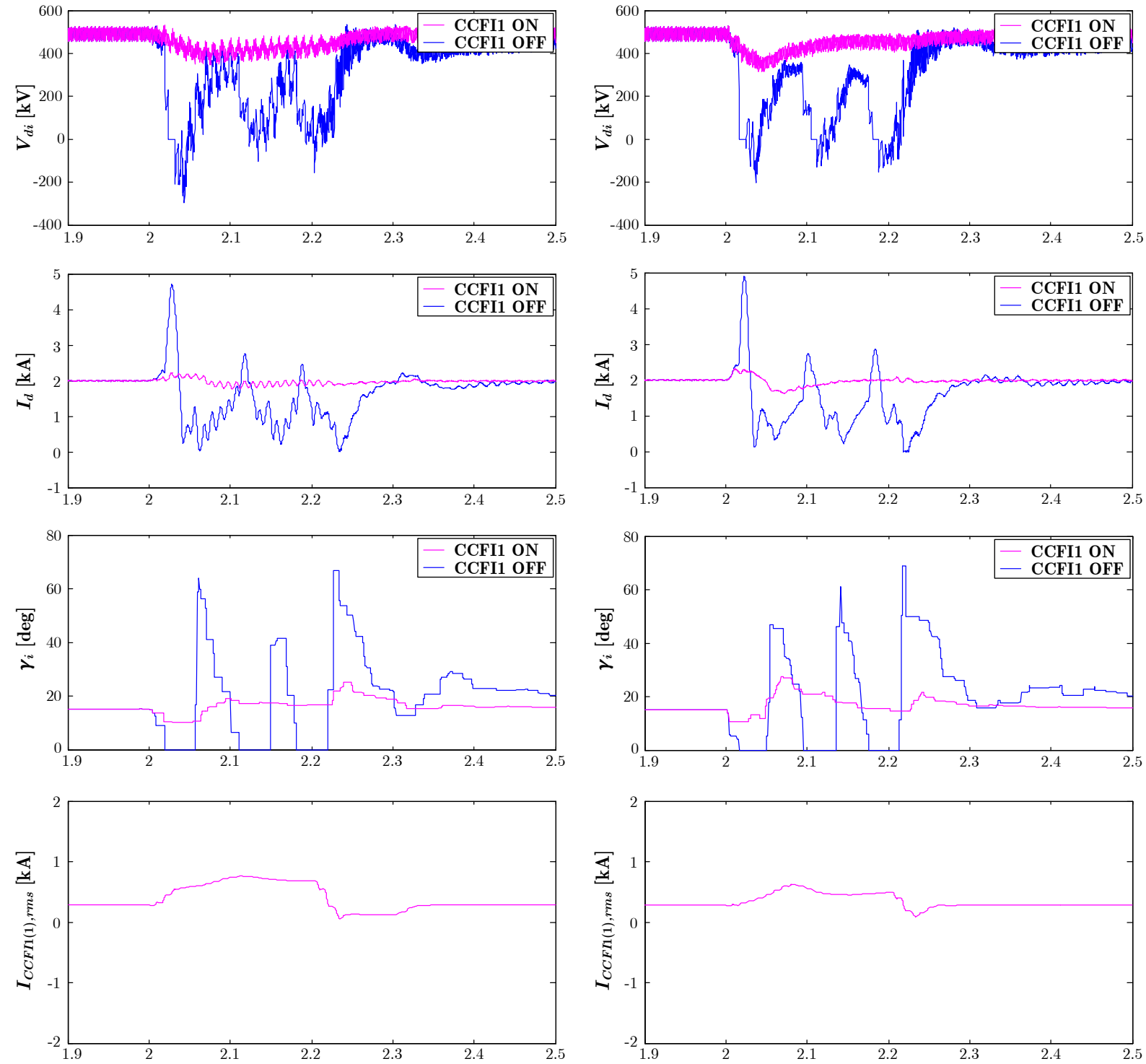

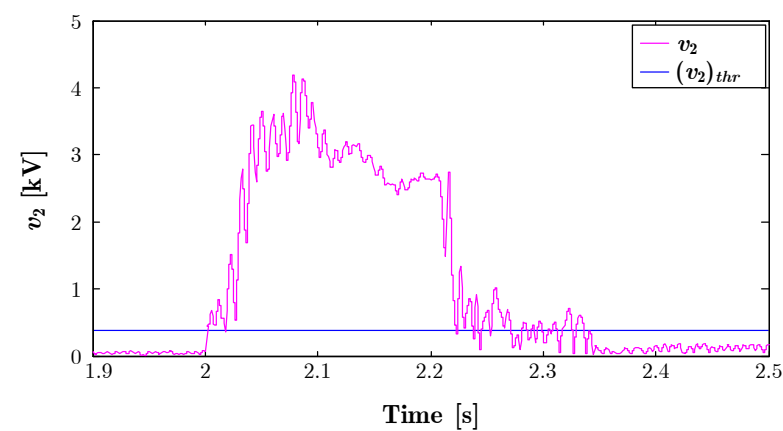

(c)

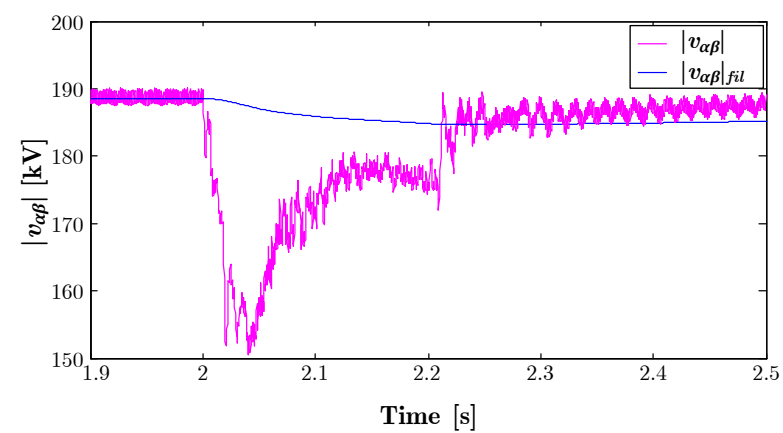

(d)

Fig. 9: The results obtained from the experimental test of the proposed CCFI under different fault types: (a) single-line-to-ground fault, (b) double-line fault, (c) double-line-to-ground fault, and (d) three-phase fault. 
capability of the inverter ac lines due to the presence of the series capacitance. In case of a fault incident at one of the inverter ac systems, its corresponding CCFI switches to the fault current limiting mode and inhibits the commutation failure. The salient feature of the developed CCFI is that it is fully controllable and has a non-complex structure and control-circuit. Besides, it neither causes excessive voltage stress on the inverter switches nor any voltage drop on the commutation circuit. To verify the effectiveness of the proposed strategy under real-time conditions, several fault cases were experimentally tested. The results showed that the developed CCFI can effectively prevent successive commutation failures at the inverter station.

\section{REFERENCES}

[1] G. Li, J. Liang, T. Joseph, T. An, J. Lu, M. Szechtman, B. R. Andersen, and Q. Zhuang, "Feasibility and reliability analysis of LCC DC Grids and LCC/VSC hybrid dc grids," IEEE Access, vol. 7, pp. 22 445-22 456 , 2019.

[2] Y. Xue, X. Zhang, and C. Yang, "Series capacitor compensated ac filterless flexible LCC HVDC with enhanced power transfer under unbalanced faults," IEEE Transactions on Power Systems, vol. 34, no. 4, pp. 3069-3080, July 2019.

[3] X. Li, C. Liu, and Y. Lou, "Start-up and recovery method with LCC-HVDC systems participation during ac/dc system black-starts," IET Generation, Transmission Distribution, vol. 14, no. 3, pp. 362-367, 2020.

[4] J. Chen, Q. Wang, and X. Zhu, "Evaluation of commutation failure risk for HVDC caused by harmonic voltage," in 2019 IEEE 3rd Conference on Energy Internet and Energy System Integration (EI2), 2019, pp. 248252.

[5] Z. Wei, Y. Yuan, X. Lei, H. Wang, G. Sun, and Y. Sun, "Direct-current predictive control strategy for inhibiting commutation failure in HVDC converter," IEEE Transactions on Power Systems, vol. 29, no. 5, pp. 2409-2417, Sept 2014.

[6] S. Tamai, H. Naitoh, F. Ishiguro, M. Sato, K. Yamaji, and N. Honjo, "Fast and predictive HVDC extinction angle control," IEEE Transactions on Power Systems, vol. 12, no. 3, pp. 1268-1275, Aug 1997.

[7] C. V. Thio, J. B. Davies, and K. L. Kent, "Commutation failures in HVDC transmission systems," IEEE Transactions on Power Delivery, vol. 11, no. 2, pp. 946-957, Apr 1996.

[8] S. Mirsaeidi and X. Dong, "An enhanced strategy to inhibit commutation failure in line-commutated converters," IEEE Transactions on Industrial Electronics, vol. 67, no. 1, pp. 340-349, Jan 2020.

[9] C. Guo, Y. Liu, C. Zhao, X. Wei, and W. Xu, "Power component fault detection method and improved current order limiter control for commutation failure mitigation in HVDC," IEEE Transactions on Power Delivery, vol. 30, no. 3, pp. 1585-1593, June 2015.

[10] J. Bauman and M. Kazerani, "Commutation failure reduction in HVDC systems using adaptive fuzzy logic controller," IEEE Transactions on Power Systems, vol. 22, no. 4, pp. 1995-2002, Nov 2007.

[11] Y. Z. Sun, L. Peng, F. Ma, G. J. Li, and P. F. Lv, "Design a fuzzy controller to minimize the effect of HVDC commutation failure on power system," IEEE Transactions on Power Systems, vol. 23, no. 1, pp. $100-107$, Feb 2008.

[12] A. Hansen and H. Havemann, "Decreasing the commutation failure frequency in HVDC transmission systems," IEEE Transactions on Power Delivery, vol. 15, no. 3, pp. 1022-1026, July 2000.

[13] S. Mirsaeidi, X. Dong, D. Tzelepis, D. M. Said, A. Dyśko, and C. Booth "A predictive control strategy for mitigation of commutation failure in LCC-based HVDC systems," IEEE Transactions on Power Electronics, vol. 34, no. 1, pp. 160-172, Jan 2019.

[14] W. Yao, C. Liu, J. Fang, X. Ai, J. Wen, and S. Cheng, "Probabilistic analysis of commutation failure in LCC-HVDC system considering the CFPREV and the initial fault voltage angle," IEEE Transactions on Power Delivery, pp. 1-1, 2019.

[15] Y. Xue, X. P. Zhang, and C. Yang, "Elimination of commutation failures of LCC HVDC system with controllable capacitors," IEEE Transactions on Power Systems, vol. 31, no. 4, pp. 3289-3299, July 2016.

[16] R. Bunch and D. Kosterev, "Design and implementation of ac voltage dependent current order limiter at Pacific HVDC Intertie," IEEE Transactions on Power Delivery, vol. 15, no. 1, pp. 293-299, Jan 2000
[17] C. W. Taylor and S. Lefebvre, "HVDC controls for system dynamic performance," IEEE Transactions on Power Systems, vol. 6, no. 2, pp. 743-752, May 1991.

[18] M. Jafar and M. Molinas, "Effects and mitigation of post-fault commutation failures in line-commutated HVDC transmission system," in 2009 IEEE International Symposium on Industrial Electronics, Seoul, South Korea, July 2009, pp. 81-85.

[19] F. Karlecik-Maier, "A new closed loop control method for HVDC transmission," IEEE Transactions on Power Delivery, vol. 11, no. 4, pp. 1955-1960, Oct 1996.

[20] M. O. Faruque, Y. Zhang, and V. Dinavahi, "Detailed modeling of CIGRE HVDC Benchmark system using PSCAD/EMTDC and PSB/SIMULINK," IEEE Transactions on Power Delivery, vol. 21, no. 1, pp. 378-387, Jan 2006.

[21] H. I. Son and H. M. Kim, "An algorithm for effective mitigation of commutation failure in high-voltage direct-current systems," IEEE Transactions on Power Delivery, vol. 31, no. 4, pp. 1437-1446, Aug 2016.

[22] T. Gao and X. Ma, "Comparison of CCC and LCC in HVDC system," Energy Procedia, vol. 16, no. 1, pp. 842-848, Jan 2012.

[23] D. Jovcic, "Thyristor-based HVDC with forced commutation," IEEE Transactions on Power Delivery, vol. 22, no. 1, pp. 557-564, Jan 2007.

[24] H. Lee, G. T. Son, J. Yoo, and J. Park, "Effect of a SFCL on commutation failure in a HVDC system," IEEE Transactions on Applied Superconductivity, vol. 23, no. 3, pp. 5600 104-5600 104, June 2013.

[25] L. Chen, H. Pan, C. Deng, F. Zheng, Z. Li, and F. Guo, "Study on the application of a flux-coupling-type superconducting fault current limiter for decreasing HVDC commutation failure," Canadian Journal of Electrical and Computer Engineering, vol. 38, no. 1, pp. 10-19, winter 2015.

[26] S. Mirsaeidi, X. Dong, and D. M. Said, "A fault current limiting approach for commutation failure prevention in LCC-HVDC transmission systems," IEEE Transactions on Power Delivery, vol. 34, no. 5, pp. 2018-2027, Oct 2019.

[27] J. Liu, Q. Liu, R. Jiang, L. Zhang, and R. Wang, "Analysis of commutation failure of HVDC transmission system with wind farm," in 2018 2nd IEEE Conference on Energy Internet and Energy System Integration (EI2), 2018, pp. 1-6.

[28] Z. Ren, J. Li, J. He, Y. Sun, and Y. Liu, "Study on fault current suppression and commutation failure in inverter side of $\pm 1100 \mathrm{kV}$ DC system," in 2018 IEEE International Conference on High Voltage Engineering and Application (ICHVE), 2018, pp. 1-4

[29] Y. Lu, H. Zhang, Y. Cao, C. Ma, D. Yang, and H. Ma, "A control strategy for suppressing HVDC continuous commutation failure risk under weak AC state," in 2019 IEEE 3rd International Conference on Green Energy and Applications (ICGEA), 2019, pp. 35-39.

[30] E. M. J, Reactive Power Control in Electric Systems. John Wiley and Sons, 1982 . 